\title{
Gestión ambiental para minimizar la contaminación de la biodiversidad en los pueblos lacustres Ciénaga Grande de Santa Marta Magdalena-Colombia
}

\section{Environmental management to minimize the contamination of biodiversity in the lacustrine villages Ciénaga Grande of Santa Marta Magdalena-Colombia}

\author{
Walter Bermúdez \\ walterbeme@hotmail.es \\ Institución Educativa Departamental Telma Rosa Arévalo. Varela \\ Colombia \\ https://orcid.org/0000-0003-4380-4247
}

Recibido: 12 de octubre del 2018

Aprobado: 05 de diciembre del 2018

\begin{abstract}
RESUMEN
El propósito general de este estudio es analizar la gestión ambiental para minimizar la contaminación de la biodiversidad en los pueblos lacustres Ciénaga Grande de Santa Marta Magdalena-Colombia. A nivel teórico, se fundamentó por autores tales como Calvo y Corraliza (2010), Gutiérrez (2013), Blanco (2012), entre otros. Desde el análisis de una investigación cualitativa, el desarrollo del estudio está en línea del método etnográfico. La información fue recabada mediante técnicas de observación directa y de entrevistas cualitativas. Como muestra se tomaron (5) sujetos, conformados por residentes de los pueblos lacustres. La fiabilidad de dicha población es relativa ya que esta es replicable, al igual se aplicara la ética como condición preponderante. A manera conclusiva se manifiesta que la Gestión ambiental demanda de una Educación Ambiental. En Colombia se requiere de procesos integradores donde el modelo pedagógico sea decisivo con el compromiso que tengan las personas en el marco de desarrollo hacia el medio ambiente, desarrollo sostenible y calidad de vida de los individuos.
\end{abstract}

Descriptores: Gestión Ambiental; contaminación; biodiversidad; educación ambiental; calidad de vida. 


\section{ABSTRACT}

The general purpose of this study is to analyze environmental management to minimize contamination of biodiversity in the Ciénaga Grande, Santa Marta Magdalena-Colombia lake villages. At the theoretical level, it was based on authors such as Calvo and Corraliza (2010), Gutiérrez (2013), Blanco (2012), among others. From the analysis of qualitative research, the development of the study is in line with the ethnographic method. The information was collected through direct observation techniques and qualitative interviews. As sample was taken (5) subjects, conformed by residents of the lacustrine towns. The reliability of this population is relative since it is replicable, as will ethics as a preponderant condition. In a conclusive way, it is stated that Environmental Management demands an Environmental Education. In Colombia, integrative processes are required where the pedagogical model is decisive with the commitment that people have in the development framework towards the environment, sustainable development and quality of life of individuals.

Descriptors: Environmental Management; pollution; biodiversity; environmental education; quality of life.

\section{INTRODUCCIÓN}

La pérdida de biodiversidad conlleva graves consecuencias para la humanidad, porque reduce en gran medida la capacidad de los ecosistemas de proporcionar los bienes y servicios que generan beneficios económicos, culturales, de salud pública, agrícolas, otros. Dentro de los beneficios que brindan los ecosistemas también están el reciclaje de nutrientes, filtración del agua y el aire, absorción de la contaminación, bancos genéticos, estética, recreación y los hábitats de la vida silvestre. Aunque es una tarea compleja asignarle un valor monetario a la diversidad biológica, no cabe duda del enorme valor que posee.

Sin embargo, a pesar de su valor biológico y económico, la problemática ambiental tiene una magnitud a nivel mundial, que compromete a todo el planeta tierra, es así como se habla permanentemente de su contaminación, la cual afecta todos los ecosistemas, dejando como consecuencia el daño ambiental; que en ocasiones es irreversible, este problema, está estrechamente relacionada con la humanidad, con sus 
formas de vida y con la manera en que desarrollan sus actividades económicas, sociales, políticas y culturales.

De tal manera se reconoce la contaminación de la biodiversidad como un espacio único y de especial vulnerabilidad e interés que describe unos determinados valores culturales, históricos, paisajísticos; un espacio acotado y frágil en el que los aspectos de interrelación y/o interdependencia de los usos y las actividades que soporta, resultan clave al estar conectadas y comprometidas su capacidad, calidad e integridad.

Del mismo modo puede señalarse que en la actualidad se manejan los siguientes datos respecto a la contaminación en Colombia, la cual muestra lo alarmante de la situación que se está viviendo en estos momentos el $16 \%$ de nuestra tierra ya es infértil, esto es que el $80 \%$ de la región andina se encuentra totalmente erosionada, siendo las regiones del Caribe, Meta, Arauca y Vichada son las zonas más golpeadas por esa contaminación en el país.

De allí que la contaminación de la biodiversidad en los pueblos lacustres Ciénaga Grande de Santa Marta Magdalena-Colombia genera problemas en este sistema dinámico de dimensiones biofísicas como el agua, aire, junto a la flora y la fauna; de dimensiones antropológicas como las características sociales, económicas, históricas, culturales e institucionales, todos ellas bajo un sistema de administración y de gobierno específico y sectorial. Estas dimensiones se encuentran interrelacionadas y en un determinado equilibrio de manera tal que al afectar a uno de ellas, se produce el inmediato desbalance en el sistema general.

Existe así, una fragmentación en la visión de este peculiar territorio con aportes parciales en su ordenación. La Ciénega, se encuentra entre la transformación por parte del hombre y la necesidad obvia de su conservación. Al parecer las preocupaciones y acciones en la ordenación del territorio, la planificación urbana y el planeamiento sectorial no logran coincidir siempre en una ordenación coherente para estos pueblos paraninfos. Cuando la unidad geográfica difiere de la realidad administrativa se genera una percepción fragmentada de los problemas; la visión del planeamiento tradicional, 
litoral, hídrico o sectorial solo abarca una parte del asunto, en la especificidad de su parcialidad se deja las cuestiones que no aborda porque no son de su competencia directa.

Asimismo, entre tantos otros flagelos que aquejan a esta comunidad, los entes gubernamentales y municipales que son los responsables directos de estos problemas, parecen no darse cuenta de tal situación, es por ello, que se analizara la problemática ambiental en cuanto a la contaminación, como tema principal que genera gran impacto en la biodiversidad en esta ecoregión y Colombia por ser un país diverso en distintos ámbitos, entre ellos el ambiental, no es ajeno a estas problemáticas. Teniendo en cuenta además, que en los últimos años el país ha desmejorado en calidad ambiental con un ritmo muy acelerado.

\section{PROCESO TEÓRICO}

A los alcances de un mayor conocimiento del presente artículo, se desplegó un resumen del trabajo, que constituye su sustentación teórica y que se estructuró de la manera seguida:

\section{Gestión ambiental}

La gestión ambiental es un proceso que permite facilitar la convivencia con el entorno que nos rodea, así como del proceso histórico que ha llevado a su deterioro actual, esta tiene como propósito que cada individuo cree conciencia de su responsabilidad, uso y mantenimiento del ambiente. Desde la visión de Calvo y Corraliza (2010), la gestión ambiental propone una nueva forma de manejar el uso adecuado de los recursos naturales, permitiendo aumentar los conocimientos, entonces surge como reflexión el promover una mejora en la calidad de vida, que a su vez sea conducente a las acciones a favor del ambiente.

En relación a esta perspectiva, la gestión ambiental, se convierte en un proceso conveniente desde todo punto de vista, que intenta mejorar las interacciones entre los 
seres humanos y el entorno, a través de conocimientos de diferentes ramas de la ciencia, asumiendo así una postura de responsabilidad y conocimiento dirigido a la sociedad, con principios éticos, que permita lograr que los individuos no vivan ajenos al contexto donde habitan.

Es posible pensar entonces, que parte de la solución de la problemática que se presenta a nivel ambiental, está en el manejo adecuado de los recursos, desde la visión de que la gestión de estos recursos naturales que son cambiantes y de soluciones factibles, se considera la concientización y sensibilización, entonces estos pasaran a ser el motor que impulsan profundos cambios.

Para, Gutiérrez (2013), la Gestión Ambiental, es una actividad dirigida a despertar conciencia, logrando modificar actitudes, valores y conductas en la sociedad, para el uso racional y la protección de la naturaleza, considerando está como su ámbito, donde los recursos son necesarios para el desarrollo integral, orientada hacia la búsqueda de una mejor calidad de vida.

Concretando, la gestión ambiental persigue continuar y asegurar el desarrollo, al tiempo que preserva, protege y conserva los sistemas vitales para el planeta, operando como una herramienta por medio de donde se inserta la variable ambiental en los procesos requeridos para mejorar la situación, buscando implantar en las personas la conciencia e importancia de la conservación de un entorno sano, así como la participación activa de las comunidades en su mejoramiento.

Desde la visión de Blanco (2012), la gestión ambiental, es inminentemente un servicio para la acción, ya que, actúa acrecentando la conciencia acerca del impacto de la actividad humana sobre el medio, con el fin de mejorar las capacidades y contribuir a las posibles soluciones a los problemas que se presentan debido al deterioro ambiental.

Se puede precisar, que con la aplicación de un sistema adecuado de gestión ambiental, se lograría mejorar las complejas relaciones e interacciones del hombre con el ambiente, de esa manera prevenir los problemas que los afectan a ambos, los 
cuales provocan un deterioro en su calidad de vida, para así lograr bienestar de las comunidades que participan en las acciones y estrategias propuestas.

Para, García y Rosales (2010), la gestión ambiental tiene sus cimientos en los elementos que la integran los cuales se encuentras conectados, desde un enfoque interdisciplinario, por esto, no puede verse como partes distintas, sino como una acción capaz de integrar procesos de gestión. De acuerdo a esto, la gestión ambiental es una poderosa herramienta que integra a los demás procesos, que no puede abordarse de manera individual, sino por medio del desarrollo de competencias de acción colectiva. En la misma línea teórica, Marconi (2014), señala que se trata de un proceso dirigido a toda la población con el propósito de incentivar y sensibilizar de manera que se pueda lograr una conducta favorable en pro de la preservación y cuidado ambiental, propiciando la participación de todos para concretar una solución que beneficie al colectivo, que debe ser orientada a la conservación de biomas o diversidad biológica, así como tomar en cuenta la dimensión sociocultural, económica y política.

A razón de los planteamientos anteriores, la gestión ambiental supone una innovación profunda, donde el éxito de la misma exige la máxima participación de las instancias del gobierno, así como de los entes encargados de velar por la protección ambiental, alcanzando experiencias que sean en sí mismas enriquecedoras y funcionales, propiciando la reflexión y participación colectiva, de esta manera que la realidades medioambientales cambie, siendo adecuada a la conciencia y pertenencia del entorno. Por su parte, Martínez (2013), señala que esta gestión respecto al ambiente, resulta vital para comprender las relaciones que existen entre los sistemas naturales y sociales, así como para entender desde una visión más holística la importancia de los diferentes factores que forman parte del génesis de los problemas ambientales. De aquí, que la adquisición de valores, la convivencia y los comportamientos son los que van a favorecer la participación efectiva de la ciudadanía en el proceso de toma de decisiones asertiva.

La gestión ambiental, debe ser entonces un factor estratégico que indica el modelo de 
desarrollo, sostenibilidad y la equidad de los propósitos, por tanto, se constituye como una herramienta que persigue mejorar las relaciones hombre - medio, con una verdadera formación social y ética, referida al entorno natural y con la finalidad de la sensibilización; de manera que todos asuman la responsabilidad de los procesos, deben darse de manera continua y permanente para facilitar la dinámica entre el individuo y el medio ambiente, relación esta que posee múltiples dimensiones, promoviendo cambios cualitativos y cuantitativos desde la personalidad del mismo.

Considerando todos los planteamientos anteriores, constituye un acierto la implantación de un programa de gestión ambiental, con el objetivo de que el individuo se convierta en un ciudadano conservador y promotor del ambiente, asumiendo una postura más digna frente al entorno, asumiendo una actitud que permita fecundar y fortalecer la visión ecológica y ambientalista, en función a esto, que ayude a utilizar sustentablemente el desarrollo de los recursos naturales, así como también proporcionar a las actuales generaciones los beneficios de tales acciones, evitando así conductas depredadoras.

\section{La gestión ambiental en Colombia}

De acuerdo con Guillén (2008 p. 106) "la Gestión Ambiental se concibe como una dimensión que debe integrarse en las propuestas gubernamentales dirigidas a la sociedad", la UNESCO, World Conference on Education for Sustainable Development (2010) plantea que en la gestión ambiental se deben reconocer valores, fomentar actitudes y aptitudes, con el propósito de mejorar y preservar las interrelaciones entre el hombre, la cultura y el medio.

En Colombia se han venido introduciendo acciones de gestión ambiental a partir de los años 70 desde la conferencia de Estocolmo, donde el propósito fue preparar al ser humano para el desarrollo, desde una mirada de protección al medio ambiente. En este sentido, "entre 2002 y 2010, en el marco de la Política Ambiental del Plan de Desarrollo: 'Cambio para construir la paz', el Ministerio del Medio Ambiente diseña y 
pone en ejecución el Proyecto Colectivo Ambiental, el cual se posiciona como su carta de navegación" (MINAMBIENTE, MINEDUCACIÓN, 2010). Este proyecto determina acciones encaminadas a generar, en la ciudadanía, criterios de ética, responsabilidad, conocimiento, así como la capacidad para identificar y enfrentar solución de los problemas ambientales, dándole vital importancia a la participación.

Muchos han sido los debates que se han dado en Colombia frente al tema de la gestión ambiental; sin embargo, desde la concepción política y desde los tomadores de decisiones, es pertinente discernir que si en Colombia se llevan casi veinte años desarrollando iniciativas para la gestión ambiental, por qué no se cuenta con un cambio social frente al medio ambiente, además cómo se han enfocado las acciones hacia la gestión ambiental; cuáles son los obstáculos que existen para formar los nuevos ciudadanos que requiere el país (Torres, 1998).

En relación a esto, Torres (1998) señala que no se tenían respuestas claras sino supuestos e hipótesis, sin embrago, tomando en cuenta el propósito que se pretendía lograr, se pensó que lo más urgente y adecuado no era la formulación de un plan nacional de Gestión Ambiental sino, conocer el país e intentar comprender cómo piensa su gente, cómo actúa, cómo desarrolla sus procesos, qué conceptualizaciones manejan, qué estrategias y metodologías se construyen.

Esta reflexión conduce a que no se puede pensar en una gestión ambiental encaminada a desarrollar acciones que conlleven al cumplimiento de indicadores nacionales, sino que está se debe construir desde un pensamiento integral que considere las particularidades de las personas; que no se conciba solo desde actividades particulares, sino que debe ser una gestión pensada desde las concepciones culturales, familiares; pero, sobre todo en Colombia, se empezó a pensar en que lo importante era que hubiera un sentido de pertenencia por su entorno, por parte de las comunidades, ya que mientras que no exista una participación comunitaria en los proyectos ambientales ni sentido de apropiación hacia sus ecosistemas, no se podrá pensar en tener un proceso de transformación en el país. 
De acuerdo con lo anterior, el programa de gestión ambiental para Colombia debe ser concebido en etapas, iniciando con la exploración, en la que se identifican los actores comprometidos con el tema; una etapa de profundización, donde se hace toda la planeación de la gestión ambiental; otra etapa de proyección, donde se busca a través de la concertación llegar a acuerdos efectivos de gestión ambiental para el país, y una etapa de estrategias, en la que se definen los lineamientos y criterios sobre los cuales se debe abordar la gestión ambiental.

Sin embargo, a pesar de los esfuerzos que se han realizado, en muchas ocasiones, en una estrategia activista en la cual no necesariamente participa toda la comunidad, sino que se centra en proyectos concretos donde participan no más que el personal encargado, dejando de lado los demás actores del proceso de gestión, por otro lado, estos proyectos ambientales se han dirigido especialmente a dar soluciones a problemáticas asociadas con temas específicos, dejando de lado el abordaje de las demás problemáticas ambientales que no se tienen en cuenta, bien por el desconocimiento de su existencia o por el facilismo de cumplir con los indicadores de gestión.

En este sentido, los procesos de gestión ambiental; requieren de la aplicación de estrategias, las cuales deben ser muy fortalecidas para que se tengan verdaderos procesos de transformación e impacto que aseguren la participación de todos los actores y se inicie con un verdadero proceso de apropiación de la necesidad de entender la problemática ambiental actual y buscar soluciones a esta.

\section{Indicadores de desarrollo sustentados en la gestión ambiental}

Los indicadores de desarrollo en cuanto a la Gestión Ambiental, según Quiroga (2007), corresponden a aquellos que dan cuenta del complejo fenómeno ambiental, desde donde sectores productivos como la minería, forestal, agricultura, o bien desde un determinado contexto, se suman a la lucha ambiental, conteniendo variables de contaminación y de recursos naturales. 
Algunos de estos indicadores ambientales son la cobertura boscosa del territorio, de contaminación de agua por coliformes, deforestación, desertificación o de cambio de uso de suelo, calidad del aire de una determinada ciudad. Aun cuando estos parezcan parciales, porque no es explícita su relación con las dinámicas socioeconómicas, son definitivamente necesarios, ya que abarcar ésta dimensión implica diseñar e implementar indicadores ambientales que probablemente antes no existían en el país, lo que permite avanzar hasta un nivel de calidad similar a brindan los indicadores económicos y sociales, que han sido primeramente instalados en los países.

Gracias al progresivo desarrollo de las políticas ambientales, los indicadores de gestión en este particular han sido, tomados en cuenta de alguna manera como parciales, por ello, es importante seguir perfeccionando de estos indicadores, considerando que son imprescindibles para alimentar la dimensión ambiental de los indicadores que se seguirán generando.

En este sentido, seguir diseñando e implementando indicadores de sostenibilidad constituye un reto mucho mayor, incluso que trasciende generaciones, tomando en cuenta que deben tener un sentido transversal o sinérgicos, que permitan tener un acceso más rápido a los significados del mundo, donde se incorpore lo económico, social y ambiental, ya que no se trata de tomar indicadores de diferentes ámbitos y ponerlos juntos pretendiendo que constituyan un sistema, así como tampoco se trata de agregarlos a través de índices comunes de medición.

En sí, de lo que se trata es poder brindar resultados desde el progreso, utilizando un número limitado de indicadores vinculantes verdaderamente, que tengan incorporados las potencialidades, sinergia, dimensiones y sectores desde su origen. Todo esto, corresponde a un desafío en el que convergen gigantescas iniciativas en el mundo, realizándose desarrollos científicos impactantes, al mismo tiempo en que se evalúa el diseño de la eficacia de las políticas públicas, por lo que su valor aumenta.

La Organización para la Cooperación y el Desarrollo Económico - OECD (2010) define un indicador ambiental como un parámetro o el valor resultante de un conjunto de 
parámetros, que ofrece información sobre un fenómeno. Así como los indicadores económicos o sociales, los indicadores ambientales están basados en series de datos específicos 0 series estadísticas que miden algunos componentes, procesos 0 tendencias de interés, que son básicamente el deterioro ambiental o la insostenibilidad; pero lo difícil es definir qué es desarrollo sostenible, o cuál es el ideal de medio ambiente o balance ecológico.

Los indicadores de gestión ambiental han adquirido relevancia en los últimos años, por su capacidad de generar una imagen sintética de las condiciones ambientales del territorio. Su auge se ha desarrollado de forma paralela a los avances, acuerdos y retos ambientales a nivel global. Lo anterior también ha conducido a la necesidad creciente de contar con información que facilite la formación de una opinión a la hora de tomar decisiones públicas o privadas, a través de la formulación de políticas ambientales para la asignación de recursos a esta área. Estos aparecen cargados de una urgencia política y de unos desarrollos informáticos que los hacen más factibles de construir que otro tipo de indicadores que han tenido mayores dificultades en su conceptualización y construcción

Entre los problemas para formular y consolidar indicadores de gestión ambiental concretos, está el que continuamente sigan en desarrollo y discusión, además, como las condiciones ambientales, sociales y políticas de cada país, región y localidad son diferentes, hay indicadores nuevos que pueden ser más importantes para un sitio específico, y a nivel general no tienen tanta importancia. Así mismo aún está en proceso la recolección, sistematización y centralización de la información requerida en muchos países.

Cabe decir que en Colombia, así como en alguna otras partes del mundo, aún se está en los principios del diseño, construcción, implementación y evaluación de indicadores, ya que se trabaja de manera simultánea con el desarrollo sostenible, al mismo tiempo se reconoce la necesidad de avanzar, en forma cooperativa y horizontal, en el desarrollo de la tercera generación en el tercer milenio. En el ámbito ambiental existen 
diferentes tipos de indicadores según la orientación y el alcance del estudio en el cual estén enmarcados. De esta manera, se pueden definir los siguientes tipos de indicadores (MIN. AMBIENTE, 2009):

Indicadores Biofísicos: Están orientados hacia el estudio de las condiciones naturales (atmosféricas, geosféricas, etc.), los recursos naturales, los ecosistemas y las funciones ecosistémicas de un territorio, que en su conjunto se asocian a su oferta natural o "patrimonio natural".

Indicadores Ambientales: Su objeto de estudio trasciende el de los indicadores biofísicos, en tanto que considera aspectos o problemas resultantes de la interacción entre el sistema sociocultural y el patrimonio natural. Este tipo de indicadores cobija temáticas propias de los hábitos y modos antrópicos de producción y consumo, tales como demanda y uso de recursos naturales, generación y aprovechamiento de residuos sólidos y líquidos (locales y globales), contaminación acústica, las tecnologías y los tipos de energía utilizados en la producción de bienes y servicios (industriales y agropecuarios) y sus problemas concomitantes (cambio climático, adelgazamiento de la capa de ozono, entre otros). De igual forma se consideran como indicadores ambientales los asociados con la gestión ambiental orientada hacia el uso racional de los recursos y del medio ambiente: conservación in-situ y ex-situ, educación e investigación ambiental, entre otros.

Indicadores de Sostenibilidad Ambiental. Estos indicadores son los mismos que fueron definidos como "ambientales", pero potenciados con un valor agregado tendiente a establecer y monitorear la sostenibilidad de la relación hombre-naturaleza. Para ello, pueden utilizar variados parámetros de comparación y contraste para monitorear y evaluar la evolución de sus valores en el tiempo (lecturas reales o por modelación), como los siguientes: capacidad de carga de los ecosistemas, resiliencia 0 capacidad de dilución de una corriente o los estándares o valores fijados nacional o 
internacionalmente, como referentes válidos de un "uso sostenible" o de una gestión adecuada de los recursos naturales y del medio ambiente en general.

Indicadores de Desarrollo Sostenible. Este tipo de indicadores dan cuenta de las cuatro dimensiones tradicionalmente asociadas al concepto de desarrollo sostenible: la ambiental, la económica, la social y la institucional. No obstante, debe existir un amplio acuerdo en que éstas son las dimensiones que se deben incluir en la definición y medición del desarrollo sostenible, no existe aún un camino suficientemente compartido para identificar y modelar las múltiples y complejas interrelaciones entre ellas y generar a partir de ellas una expresión sintética y agregada que exprese el avance obtenido en la construcción del desarrollo sostenible.

\section{JUSTIFICACIO METODOLÓGICA}

Desde una perspectiva epistemológica, la investigación cualitativa se orienta hacia la construcción del conocimiento acerca de la realidad social, cultural y ambiental a partir de la interpretación de la perspectiva de los sujetos involucrados. Tal postura metodológica, implica asumir un carácter dialógico en la creencia, las mentalidades, los mitos, perjuicios y sentimientos, todos los cuales son aceptados como elemento de análisis para producir conocimiento sobre la realidad humana.

A razón de lo anterior, Rojas (2007) señala que problemas tales como descubrir el sentido, la lógica y la dinámica de las acciones concretas, se convierten en una constante de las perspectivas cualitativas. Desde lo ontológico, el manejo de la realidad o de algunos de sus componentes es imprescindible para construir o asumir definiciones, al pensar en la realidad implica transformar en conjunción, entre la reflexión y la acción, desde el conocimiento propio.

Desde este punto de vista cualitativo, la investigación se desarrolla con el método etnográfico, definido por Hernández, Fernández y Baptista (2014), como el estudio que pretende investigar grupos que comparten una cultura, en este particular los pueblos 
lacustres de la Ciénaga Grande de Santa Marta, en Colombia, para lo cual es el investigador quien selecciona el lugar, realiza la detección de sus informantes para luego recolectar y analizar los datos obtenidos, esto se traduce, que la importancia de que este tipo de estudio está en describir, y analizar ideas, significados, conocimientos, de un grupo selecto de individuos.

En correspondencia con lo anterior, está investigación considera el propósito de los estudios etnográficos, por cuanto describe y analiza el fenómeno que se desarrolla de diseñar un programa de gestión ambiental para minimizar la contaminación de la biodiversidad en los pueblos lacustres Ciénaga Grande de Santa Marta MagdalenaColombia.

Por lo que los informantes clave que se eligen pueden emitir su opinión de acuerdo a este planteamiento, para luego dejar en los resultados reflejadas las situaciones que se desarrollan, cumpliendo con los propósitos del estudio para finalmente diseñar un programa de gestión ambiental ajustado a la realidad que esos pueblos viven, convirtiéndose en un aporte del estudio al progreso de la investigación científica.

\section{DERIVACIONES CONCLUSIVAS DE LA INVESTIGACIÓN}

La Gestión ambiental demanda de una Educación Ambiental. En este sentido en Colombia se requiere de procesos integradores donde el modelo pedagógico sea decisivo en el compromiso que tengan las personas en el marco de desarrollo hacia el medio ambiente, desarrollo sostenible y calidad de vida los individuos.

Fundamentado en el criterio del pensar, aprender y actuar y de esta manera generar una cultura Ambiental. Como resultado de todo proceso investigativo la gestión ambiental debe ir dirigida a la solución de la problemática ambiental teniendo en cuenta la relación que existe entre las personas y el entorno que no se limita únicamente a considerar el entorno como el espacio físico donde se da una conducta determinada, sino a comprender dicha relación a partir de que es el espacio, quien va a imprimir al individuo ciertos significados para llevar una interacción constante, en 
donde los actores interpretan y elaboran nuevas construcciones, con el fin de acrecentar las bases de una identidad social afiliada al entorno, determinado a partir de:

- Implementar la propuesta alternativa para el desarrollo de una Gestión Ambiental en Colombia, para dinamizar el proceso de la contaminación de la biodiversidad en los pueblos lacustres y así una mayor reciprocidad en cuanto al Medio Ambiente y la comunidad.

- Propiciar un modelo práctico y responsable para una educación ambiental, dinámica y participativa que facilite el desarrollo de una cultura ambiental.

En este sentido la cultura, ambiental es una corriente internacional de pensamiento y acción, su meta es procurar cambios individuales y sociales que provoquen la mejora ambiental y un desarrollo sostenible, proporcionando la información y los conocimientos necesarios en la población colombiana para que esta adquiera conciencia de los problemas del ambiente, creando en ella predisposición, motivación, sentido de responsabilidad, pertenencia y compromiso para trabajar individual y colectivamente en la búsqueda de soluciones, lo cual va forjando una identidad ambiental orientada hacia la construcción de significados, expresados en como los recursos naturales representan una calidad de vida, la satisfacción de necesidades básicas y fortalecer la relación con su entorno.

Asimismo, se busca proporcionar una educación ambiental que se centre en la relación hombre - entorno y que sea abordada desde la disciplina de pedagogía que pretende mejorar los procesos de enseñanza - aprendizaje en todos los niveles de educación formal y la psicología ambiental, la cual permite hacer una descripción de los comportamientos que adoptan los hombres y las mujeres en relación a la utilización de los recursos naturales tales como: actividades de guardabosques, reforestación, creación de viveros, recolección de basuras entre otros. En sentido se puede decir que la población colombiana debe desarrollar acciones que permitan la participación en grupos de aprendizaje colaborativo y cooperativo que reflejan un aprovechamiento de 
las actividades agrícolas, desde las tareas que cumplen a diario.

Así como lo afirma Rengifo (2007 p.173) "El aprendizaje colaborativo tiene lugar a través de la interacción en un contexto social, en la cual los estudiantes resuelven problemas, responden preguntas, discuten, explican, debaten". Al mismo tiempo, dichas acciones permiten una adecuada planificación para el desarrollo de estrategias de conservación permitiendo que los recursos se mantengan en las regiones y con ello podemos afirmar que los estilos de vida de las personas se van estableciendo, dependiendo de la interacción que tenga cada uno con los recursos de la región.

La presente propuesta se fundamenta en la sostenibilidad de un sistema de valores que exige responsabilidad, voluntad, compromiso, planificación democrática y participativa en un marco ético de cooperación y solidaridad, capaz de superar el individualismo insolidario y competitivo. El desarrollo humano es un objetivo a perseguir, es decir un proceso de ampliación de las oportunidades, como también el nivel de bienestar alcanzado. Así mismo, pretende ayudar a distinguir entre la formación de capacidades humanas y cómo se utilizan las capacidades adquiridas como son alcanzar una vida larga y saludable, que tengan conocimientos y que cuenten con acceso a los recursos necesarios, la participación de las personas en la toma de decisiones para que sean agentes activos de su propio desarrollo.

Las personas en las comunidades deben ser cooperativas lo cual les permitirá arraigar el sentido de pertenencia a la comunidad, es decir, la cohesión social ha de estar basada en la cultura, los valores y las creencias compartidos, se debe perfilar la equidad, es decir, capacidades básicas y oportunidades de vida. La equidad implica la no discriminación por razón de género y con ello sostenibilidad lo cual implica equidad intra e intergeneracional.

Pensando en la participación debe ser un elemento esencial denominado democracia cultural activa, integral y pluridimensional de la población en el complejo proceso de construcción de su vida individual y colectiva. La democracia cultural tiene por objetivo colaborar en la realización del derecho de la humanidad a la participación en la toma 
de decisiones.

La democracia es entendida como el conjunto de relaciones socio-políticas que permiten la participación de los individuos en las decisiones de la sociedad y que aseguran las condiciones necesarias para garantizar su plena expresión y desenvolviendo. La participación es un proceso activo en el que se interpretan los planos individual y social que respete la identidad y la diversidad y la diversidad culturales, es un acto democrático y un proceso de auto aprendizaje individual y colectivo que transcurre en el propio proceso de toma de decisiones y que implica el compromiso activo de quienes deciden intervenir.

\section{REFERENCIAS CONSULTADAS}

1. Blanco, M. (2012). Gestión ambiental: camino al desarrollo sostenible. San José de Costa Rica: EUNED, Editorial Universidad Estatal a Distancia.

2. Calvo, S y Corraliza, J (2010). Educación ambiental. La Habana: Universidad de Pinar del Río "Hermanos Saíz Montes de Oca". P. 22.

3. García G., J. y Rosales, J. N. (2010). Estrategias didácticas en Educación Ambiental. Málaga (España). Ediciones Aljibe, S. L.

4. Guillén, F. C. (2008). Educación, medio ambiente y desarrollo sostenible. Revista Iberoameriana de Educación,11, 103-110. Recuperado de http://www.oei.es/oeivirt/rie11a03.pdf.

5. Gutiérrez, M (2013) El Sistema de Gestión Ambiental. Universidad El Bosque. Revista de Tecnología. Journal of Technology. Volumen 14.

6. Hernández, Fernández y Baptista (2010). Metodología de la investigación. Editorial. Mc Graw Hill México D.F.

7. López Echegarai, M., \& Benítez Álvarez, J. (2018). La Aplicación de la Evaluación de los Aprendizajes. Un estudio en la Universidad Bolivariana de Venezuela. Revista Arbitrada Interdisciplinaria Koinonía, 3(5), 67-83. Recuperado de

http://fundacionkoinonia.com.ve/ojs/index.php/revistakoinonia/article/view/115/97 
8. Marconi, R. (2014). Orden, desorden, caos: ¿un nuevo paradigma? Revista Insomnia, 3. Recuperado https://www.redalyc.org/pdf/3217/321750362021.pdf.

9. Martínez, E. H. (2013). La relación cultura-naturaleza en la arquitectura occidental. Cali: Artes Gráficas del Valle - Universidad del Valle.

10. Ministerio del Medio Ambiente (MINAMBIENTE) y Ministerio de Educación Nacional (MINEDUCACIÓN). (2010). Política nacional de educación ambiental Sina.Bogotá: Autor. Recuperado de http://webcache.googleusercontent.com/search?q=cache:I1zUzPxwXtoJ:cmap.up b.edu.co/rid\%3D1195259861703_152904399_919/politi-

ca_educacion_amb.pdf $+\& c d=1 \& \overline{\mathrm{hl}}=\mathrm{e} s \& \mathrm{ct}=\mathrm{cln} \overline{\mathrm{k}}$.

11.OECD, Organization for Economic Co-operation and Development (2010), Environment at a Glance, Environmental Indicators, Paris. [http://www.oecdbookshop.org/oecd/display.asp?sf1=identifiers\&lang=EN\&st1=97 2005081p1].

12. Quiroga, R. (2007). Indicadores ambientales y de desarrollo sostenible: avances y perspectivas para América Latina y el Caribe. Santiago de Chile, Chile: Comisión Económica para América Latina y el Caribe.

13.Rengifo, B (2007) La educación ambiental una estrategia pedagógica que contribuye a la solución de la problemática ambiental en Colombia. San Juan de Pasto. XII Coloquio Internacional Geocrítica. Disponible en: https://s3.amazonaws.com/academia.edu.documents/32460823/06-BRengifo.pdf?AWSAccessKeyld=AKIAIWOWYYGZ2Y53UL3A\&Expires=15472965 47\&Signature=W5PiUuuHCN41eTMWsNUn92B7kt0\%3D\&response-contentdisposition=inline\%3B\%20filename\%3DLA.

14. Rojas, R. (2007). Guía para realizar investigaciones sociales. México: Plaza y Valdés Editores.

15. Torres, M. (1998). La educación ambiental: Una estrategia ï $\urcorner$,exible, un proceso y unos propósitos en permanente construcción. La experiencia de Colombia. Revista Iberoamericana de Educación, 16, 23-48. Recuperado de http://www.rieoei.org/oeivirt/rie16a02.pdf. 
KOINONIA. Revista Arbitrada Interdisciplinaria de Ciencias de la Educación, Turismo, Ciencias Sociales y Económica, Ciencias del Agro y Mar y Ciencias Exactas y aplicadas. Año IV. Vol IV. Nº . Enero - Junio 2019. Hecho el depósito de Ley: FA2016000010 ISSN: 2542-3088

FUNDACIÓN KOINONIA (F.K). Santa Ana de Coro. Venezuela.

Walter Bermúdez

16.UNESCO, World Conference on Education for Sustainable Development. (2008). World Conference on Education for Sustainable Development. Bonn Declaration [Conferencia Mundial sobre educación para el desarrollo sostenible. Declaración de Bonn]. Bonn, Germany: Autor. Recuperado de http://unesdoc.unesco.org/images/0018/001887/188799e.pdf

(C2019 por el autor. Este artículo es de acceso abierto y distribuido según los términos y condiciones de la licencia Creative Commons Attribution (http://creativecommons.org/licenses/by/4.0/). 